SEÇÃO TEMÁTICA: EXPERIÊNCIAS DE ENSINO SUPERIOR ALTERNATIVO

\title{
Dentro ou Além da Universidade? Experiências de ensino superior alternativo
}

\author{
Tristan McCowan' \\ Gunther Dietz" \\ 'University College London (UCL), London - Reino Unido \\ "Universidad Veracruzana, Xalapa - México
}

RESUMO - Dentro ou Além da Universidade? Experiências de ensino superior alternativo. Apesar de um importante processo de expansão e crescimento, as instituições de ensino superior em todo o mundo ainda enfrentam a exclusão, a discriminação e a desigualdade no que diz respeito às comunidades e povos sub-representados e desfavorecidos, seus conhecimentos e suas línguas. Neste texto, apresentamos brevemente alternativas contemporâneas ao ensino superior regular, que - algumas dentro das universidades existentes, outras além das estruturas convencionais de ensino superior - estão trabalhando contra a corrente para resolver essas falhas. Ao apresentar os estudos de caso das universidades alternativas, interculturais, indígenas, descoloniais, cooperativas e/ou de movimentos sociais que compõem esta seção temática, identificamos tendências institucionais e organizacionais, questões temáticas e contribuições teóricas emergentes, com o objetivo de aprofundar a compreensão da orientação e do papel dessas instituições, seus impactos e os desafios enfrentados.

Palavras-chave: Ensino Superior. Educação Alternativa. Movimentos Sociais. Pluriversidades. Subversidades.

ABSTRACT - Within or Beyond the University? Experiences of alternative higher education. Despite an important process of expansion and growth, higher education institutions around the world still reproduce exclusion, discrimination and inequality with regard to underrepresented and underprivileged communities and peoples, their knowledges and their languages. In this text, we briefly frame contemporary alternatives to mainstream higher education that - some within existing universities, some beyond conventional higher education structures - are working against the grain to address these failings. By introducing the case studies of alternative, intercultural, indigenous, decolonial, co-operative and/or social movement universities that make up this special issue, we identify institutional and organisational tendencies, topic issues and emerging theoretical contributions, thus aiming to develop a deeper understanding of the orientation and role of these institutions, their impacts and challenges faced.

Keywords: Higher Education. Alternative Education. Social Movements. Pluriversities. Subversities.

Educação \& Realidade, Porto Alegre, v. 46, n. 4, el20754, 2021. 
Dentro ou Além da Universidade?

À luz dos extremos desafios enfrentados pela humanidade no momento atual, qual resposta as instituições de ensino superior precisam dar? A mudança climática e a degradação ambiental estão ameaçando o delicado equilíbrio ecológico que tem sustentado a existência de nossa espécie no planeta e, apesar do aumento da prosperidade material para muitos, as desigualdades dentro e entre as nações estão arraigadas. A pandemia da Covid revelou a tolice do senso de invulnerabilidade humana e da separação da natureza, além de ter exacerbado a situação social e econômica (Silova, 2021). Este cenário nos convida a nos debruçarmos sobre o modelo existente de ensino superior para dotá-lo de mais efetividade para responder a estes desafios, ou esse próprio modelo está implicado em causar os problemas?

Os sistemas de ensino superior passaram por um crescimento impressionante nas últimas décadas no mundo inteiro e receberam reconhecimento renovado por seu papel no desenvolvimento social e econômico (Arnhold; Bassett 2021; Marginson, 2016). Apesar da expansão em termos de vagas, continuam a ser altamente desiguais e a excluir determinados grupos ou confiná-los em instituições de qualidade inferior (UNESCO, 2017). Além disso, os modelos de excelência promovidos por ranqueamentos internacionais limitaram a capacidade das universidades de promover o desenvolvimento inclusivo, diversificar e/ou interculturalizar seus programas de estudo, engajar-se com as comunidades locais em um diálogo respeitoso e recíproco, e promover a consciência crítica entre os estudantes (McCowan, 2019). Embora não haja dúvida de que as universidades convencionais estejam fazendo contribuições positivas à sociedade de muitas maneiras diferentes, estas limitações significam que, no mínimo, precisamos pensar na possibilidade de modelos alternativos.

Esta seção temática explora alternativas ao ensino superior convencional que estão trabalhando na contramão para responder a estas falhas. Ao incorporar estudos de caso de universidades alternativas, interculturais, indígenas, decoloniais, cooperativas e/ou de movimentos sociais em diferentes países, análises transnacionais e tratamentos teóricos, objetiva desenvolver uma compreensão mais profunda da orientação e da função destas instituições, de seus impactos e dos desafios enfrentados.

O número destas iniciativas contra-hegemônicas em prática é considerável e muitas delas estão reunidas na Aliança de Ecoversidades, discutida na contribuição de Mandel, Amaro e Teamey nesta seção temática. Estas iniciativas autônomas, situadas nas margens de sistemas formais de ensino superior, sem restrições de regulação e acreditação pelo governo, possuem considerável liberdade para experimentação e expressão de seus princípios. Entretanto, também há resistência e construção de alternativas no sistema convencional. Esta seção temática também aborda desafios às estruturas universitárias convencionais (como currículo, qualificações, requisitos para ingresso, função do docente e iniciativas de serviço comunitário) e às tradições de conhecimento convencional, abrindo a instituição para a diversidade epistêmica, linguística e/ou cultural, inclusive o pensamento indígena 
e contra-hegemônico. Estas duas formas - por um lado, bolsões de inovação em grandes instituições e, por outro, iniciativas populares fora do convencional - correspondem em grande medida à diferenciação que Boaventura de Sousa Santos $(2017 ; 2018)$ faz entre a pluriversidade e a subversidade, uma formulação mobilizada em diversas análises neste número do periódico. Por fim, existem aquelas iniciativas situadas em um espaço limítrofe, entre a corrente convencional e as margens, ou com um pé em cada uma (McCowan, 2016). Esse é o caso das iniciativas do Movimento dos Trabalhadores Rurais Sem Terra (MST) e de outras iniciativas de educação rural no Brasil, abordadas na contribuição de Schwendler e Santos.

Compreender e avaliar as alternativas atende a muitas finalidades. De uma perspectiva analítica, é importante documentar as diferentes manifestações de ensino superior e medir o impacto que as variações têm sobre os desfechos relacionados com aprendizagem, produção de conhecimento e engajamento com a sociedade. Mesmo assim, existem claras implicações normativas nesta tarefa. Conforme foi indicado acima, estas alternativas podem nos oferecer caminhos fecundos a buscar ao abordar as atuais crises ecológica e social, como os laboratórios de experimentação e de inovação. Atualmente podem ser pequenas, mas algumas intervenções bem-sucedidas podem ser expandidas para alcançar um maior número de pessoas. Como alternativa, podemos observar que parte do problema é nossa ênfase atual na uniformidade em grande escala, e o que é de fato necessário é um florescimento de inúmeras iniciativas em pequena escala que podem abordar de maneiras singulares os diferentes interesses, desafios e necessidades locais.

Fundamentalmente, as alternativas nos mostram que essa outra maneira de fazer as coisas é possível, renovando nossa vontade de experimentar e proporcionando confiança em nossa imaginação de futuros mais sustentáveis e justos. A inovação desta forma é particularmente difícil, dadas as tendências gerais do ensino superior rumo ao isomorfismo, com evidente deriva institucional, mesmo em sistemas que demonstram diversidade entre instituições técnicas e acadêmicas, abastecidas por ranqueamentos internacionais e outros incentivos à imitação (Morphew; Huisman, 2002; DiMaggio; Powell, 1983).

A literatura atual nesta área é escassa. Nos últimos anos, foi prestada significativa atenção a novos modelos de ensino superior, especificamente ao surgimento de universidades on line (como as gigantes Indira Gandhi National Open University na Índia e a Allama Iqbal Open University no Paquistão), a introdução de tecnologias digitais (Goodfellow; Lea, 2013; Williamson, 2018), o surgimento de cadeias com fins lucrativos com modelos padronizados de franquia (especialmente no Brasil e nos Estados Unidos), a desagregação dos elementos constituintes (Czerniewicz et al., 2021; McCowan, 2017), e até mesmo o surgimento de uma universidade blockchain no estilo de bitcoin ${ }^{1}$. No entanto, com poucas exceções, estas alternativas são técnicas e não políticas, oferecendo diferentes modalidades de oferta ao vincularem o ensino superior de maneira mais íntima com o modelo econômico capitalista. 
Dentro ou Além da Universidade?

Os artigos desta seção temática estão embasados em um rol de recursos teóricos. As ideias de Santos $(2017 ; 2018)$, mencionadas acima, são proeminentes. Seu trabalho sobre epistemologias do Sul e ecologia de saberes ofereceu importantes ferramentas conceituais para formular o projeto de conhecimento de universidades alternativas, e categorizaram diferentes formas de ensino superior contra-hegemônico. A tradição mais longa do pensamento anarquista também é influente em relação a isso, conforme é discutido na contribuição de Hudson-Miles.

As filosofias indígenas também influenciaram o desenvolvimento destas iniciativas, particularmente na região da América Latina, onde noções locais e regionais de sumak kawsay ou buen vivir, mas também de tapalewilis, tequio, minga ou reciprocidade, assim como de comunalidad se contrapõem aos modelos cumulativos insustentáveis do capitalismo global contemporâneo, bem como aos modelos educacionais que o sustentam. Com base nesses conceitos alternativos, indígenas e/ou populares, as experiências de indigenizar e interculturalizar as universidades estão começando a reformatar o ensino superior de cosmologias e epistemologias não ocidentais (Mateos Cortés; Dietz, 2016). Essa virada de baixo para cima representa uma tentativa acadêmica e política de decolonizar, desafiar e, assim, reinventar as importantes funções das universidades convencionais, como ensino e aprendizagem, mas também pesquisa e extensão (Santhakumar et al., 2020).

Embora usemos o termo alternativa/o nesta seção temática, estamos bem cientes de suas limitações. Chamar algo de alternativo pode subestimá-lo, consolidando sua natureza marginal em relação ao convencional estabelecido e potente, ou sinalizar que é apenas do interesse daqueles com gosto divergente ou estilo de vida excêntrico. Ao contrário, o posicionamento assumido nesta seção temática é que estas iniciativas, apesar da pequena escala, representam experiências cruciais para a humanidade como um todo. No entanto, na falta de alternativa (!), a palavra alternativa/o foi usada aqui, juntamente com um rol de outros termos como autônomo, inovador, experimental e contrahegemônico, e em alguns outros casos, especificamente indígenas ou ambientais. Fazendo um paralelo com os debates nos estudos do desenvolvimento sobre o desenvolvimento alternativo e alternativas $a$ o desenvolvimento, poderíamos questionar se estas iniciativas são ensino superior alternativo ou alternativas ao ensino superior. Alguns dos casos abordados nesta seção temática tendem mais para a primeira opção e outros para a última.

Abrimos a seção temática com um artigo conceitual abrangente de autoria de Tristan McCowan intitulado Desinstitucionalização e renovação no Ensino Superior. Iniciando com uma problematização das estruturas centrais compartilhadas por instituições de ensino superior em diferentes lugares do mundo, o autor identifica os principais aspectos destas estruturas ao diferenciar portas de entrada - os controles sobre a admissão à universidade -, funções - as diferenciações entre estudantes, docentes e outros atores - e carimbos - os processos e artefatos de validação da aprendizagem e do conhecimento do estudante. $\mathrm{O}$ arti- 
go esboça implicações para o futuro da universidade e para as iniciativas atuais que estão tentando subverter estas estruturas e criar modelos alternativos.

Em sua contribuição Aliança de Ecoversidades - Uma experiência de cinco anos em aprendizagem cosmopolitica, Udi Mandel, Gerardo Lopez Amaro e Kelly Teamey questionam como podemos reinventar o ensino superior para incluir múltiplos saberes. Como uma possível resposta, apresentam a Aliança de Ecoversidades, uma aliança planetária de lugares e profissionais de ensino que reinventam o ensino superior, muitas vezes embasados em movimentos sociais e ecológicos e em comunidades indígenas. Seu artigo analisa diversas experiências de aprendizagem cosmopolítica, de aprender a aprender em e entre culturas, epistemologias e ontologias.

Em Rumo a um sistema de ensino superior para estudantes indigenas? Universidades interculturais no México, Gunther Dietz e Laura Selene Mateos Cortés contribuem com uma análise crítica das universidades interculturais, um novo tipo de instituição de ensino superior criado por iniciativas governamentais e não governamentais em diferentes regiões indígenas do México. Os autores identificam tensões e contradições compartilhadas por estas instituições, mas também identificam diferentes tipos de universidades interculturais no que tange à variação de autonomia, sua orientação de cima para baixo ou de baixo para cima, e seus passos para plurilingualizar e interculturalizar sua práxis de ensino e aprendizagem.

Um segundo artigo sobre o México é apresentado por Germán Ortiz Palomeque. Em Intentando hacer grietas en el Sistema Educativo Mexicano: los contrapuntos en los aprendizajes de los estudiantes de la Licenciatura en Planeación del Desarrollo Rural del Centro de Estudios para el Desarrollo Rural (CESDER) en la Sierra norte de Puebla, o autor analisa o modelo alternativo de ensino superior do CESDER por meio de experiências de seus estudantes e docentes. Especificamente, a ênfase nos saberes camponeses do próprio estudante proporciona um processo de aprendizagem intimamente ligado a problemas locais e regionais, o que transforma os estudantes em agentes de transformação nas lutas de suas comunidades contra projetos de morte extrativistas efetivados por empresas transnacionais em toda a região.

Do México para o Brasil, Diana Pellegrini, Elie Ghanem e Antônio Góes Neto analisam em seu artigo O Brasil respeita o direito dos povos indígenas ao ensino superior? Demanda, oferta e ensaios alternativos em São Gabriel da Cachoeira, Amazonas como as demandas indígenas por ensino superior são oficialmente negadas, mas são oferecidas de maneira alternativa por iniciativas regionais. Ao traçar um panorama de oportunidades educacionais para populações indígenas no noroeste da Amazônia brasileira, descrevem dois cursos de formação de professores que implementam práticas alternativas, mas que ainda não refletem as demandas reivindicadas pelos povos indígenas da região. 
Dentro ou Além da Universidade?

Em seu artigo A formação de educadoras/es no contexto socioterritorial do campo, Sônia Fátima Schwendler e Aline Nunes dos Santos enfocam iniciativas de formação de professores que objetivam incluir em programas de estudos oficiais um deslocamento decolonial rumo ao reconhecimento da diversidade. Ao escolher a Licenciatura em Educação do Campo (LECAMPO) oferecida pela Universidade Federal do Paraná em uma abordagem descentralizada para alcançar comunidades camponesas, indígenas e quilombolas em suas regiões de origem, as autoras estudam empiricamente as experiências de professores e de formadores de professores com um programa itinerante e territorializador que está transformando noções importantes de formação de professores.

Do Brasil para o Chile, o artigo Hacia un vínculo decolonial universidad-escuela: problematizando nuestras microprácticas coloniales mediante cuidado epistémico, de Paulina Bravo González, Felipe Acuña Ruz, Corina González Weil, Nina Ibaceta Guerra, Roberto Morales Aguilar e Bárbara González Urzua problematiza a relação colonial corrente entre escolas e universidades, entre o conhecimento acadêmico e os atores escolares e processos educacionais. Por meio do exemplo de um centro para ensino de ciências da Pontifícia Universidad Católica de Valparaíso, identificam os primeiros passos para um tipo de ensino decolonial de ciências que reconhece e interrelaciona a pluralidade de saberes existente.

Uma segunda contribuição do Chile é o artigo de Francisco Durán del Fierro e Roxana Pey Tumanoff, Territorialidad y neoliberalismo: el caso de la Universidad Estatal de Aysén no Chile. Os autores refletem sobre a Universidad de Aysén em sua tentativa de se transformar em uma subversidade ao traduzir expectativas nacionais e locais em práticas institucionais que enraízam a universidade no território. Apesar de determinadas falhas, relacionadas à dominância de um modelo de universidade de mercado neoliberal, a criação de um conselho social que cogoverna a universidade significa um passo à frente no processo de subverter funções convencionais da universidade.

Da América Latina para a Europa, Richard Hudson-Miles faz em seu artigo Experiências em Ensino Autônomo de Artes no Reino Unido, 2010-até o presente um exame crítico das escolas de arte autônomas estabelecidas no Reino Unido no contexto de processos neoliberais de financeirização, mercantilização e comercialização do ensino superior. Inspirado pela pedagogia crítica e pela teoria educacional anarquista, o autor realiza uma copesquisa militante junto a duas escolas britânicas de antiarte - a School of the Damned em Londres e a Feral Art School em Hull - que demonstra como os experimentos de ensino superior em artes podem ser pedagogicamente repolitizados.

Do Reino Unido para a Índia, o artigo comparativo Um Entre Muitos: Instituições de ensino superior em um ecossistema de pedagogias urbanas, elaborado por Adriana Allen, Geetika Anand, Ruchika Lall e Julia Wesely, analisa como, no campo do ensino de planejamento urbano, os arranjos institucionais de ensino, prática e pesquisa inspirados pela 
teoria urbana do Sul podem contribuir para uma virada urbana em pedagogia crítica. Uma comparação de duas experiências de aprendizagem inovadoras, o Indian Institute for Human Settlements e a School of Popular Urbanism da rede da Habitat International Coalition América Latina, oferece insights para desacomodar a ideia hegemônica de universidade; com isso, reformula as instituições de ensino superior como "um entre muitos" atores e instituições que dão forma à aprendizagem e às práticas urbanas.

Por fim, a seção temática é concluída com o diálogo Experiências em Ensino Superior Alternativo: uma conversa com Boaventura de Sousa Santos e Luis Fernando Cují Llugna. Esta discussão reúne os principais tópicos tratados nos artigos, enfatizando as tensões inerentes tanto a projetos de pluriversidade como de subversidade para transformar as relações do poder, além das assimetrias linguísticas, culturais e epistêmicas por meio de iniciativas de ensino superior iniciadas dentro, nas margens ou completamente fora das universidades existentes.

As contribuições à seção temática abrangem diversos contextos - Índia, Chile, Brasil, México e Reino Unido -, além de análises globais e teóricas. O agrupamento na América Latina é em parte devido à localização e aos interesses do periódico e dos editores, mas também é um testemunho da riqueza desta região em termos de movimentos e inovações políticas e educacionais. Esta é uma seção temática multilíngue, com artigos publicados em inglês, espanhol e português: o multilinguismo é uma parte importante do pluralismo epistêmico que sustenta este trabalho, incluindo não apenas grandes idiomas globais (e anteriormente coloniais) como estes três, mas outros idiomas nacionais, locais e indígenas que raramente estão representados na academia.

Os autores desta seção temática são pesquisadores acadêmicos, mas em muitos casos estão pessoalmente envolvidos nas iniciativas em questão - mediando suas funções como narradores e cientistas sociais e, por outro lado, como participantes, profissionais e ativistas.

As ruínas da Nalanda University na Índia, com seus 700 anos de história, oferecem uma amostra da riqueza das diferentes tradições em ensino superior do mundo inteiro - dando lugar a uma homogeneização crescente, apesar de alguma diferenciação superficial (McCowan, 2019). Esta diversidade precisa ser reavivada. A universidade de pesquisa convencional e o paradigma científico ocidental em que está baseada ainda têm muito a oferecer, mas deveria estar fundada no diálogo com as muitas outras tradições de saberes do mundo, ao invés de ser proporcionada à força (Unesco, 2021). Se quisermos abraçar o pluriverso, em vez de salvar o mundo (Arora; Sterling, 2020), então precisamos de sistemas de ensino superior nacionais e globais que representem uma multiplicidade de tradições do conhecimento, ideias, idiomas e atores, bem como um espaço para o surgimento de novas formas de instituição, até então não reconhecidas.

Traduzido por Ananyr Porto Fajardo

Educação \& Realidade, Porto Alegre, v. 46, n. 4, e120754, 2021. 
Dentro ou Além da Universidade?

Recebido em 5 de agosto de 2021

Aprovado em 30 de outubro de 2021

\section{Notas}

1 https://blockchain.open.ac.uk/

\section{Referências}

ARNHOLD, Nina; BASSETT, Roberta. Steering tertiary education: towards resilient systems that deliver for all. Washington, DC: World Bank, 2021.

ARORA, Saurabh; STIRLING, Andy. Don't save 'the world' - embrace a pluriverse! STEPS Centre, Falmer, 24 Oct. 2020.

CZERNIEWICZ, Laura et al. Academics Teaching and Learning at the Nexus: Unbundling, Marketisation and Digitisation in Higher Education. Teaching in Higher Education, p. 1-15, 2021. DOI: 10.1080/13562517.2021.1876019.

DIMAGGIO, Paul J.; POWELL, Walter W. The iron cage revisited: institutional isomorphism and collective rationality in organizational fields. American Sociological Review, v. 48, p. 147-60, 1983.

GOODFELLOW, Robin; LEA, Mary (Ed.). Literacy in the digital university: Critical perspectives on learning, scholarship and technology. London: Routledge, 2013.

MARGINSON, Simon. The worldwide trend to high participation higher education: Dynamics of social stratification in inclusive systems. Higher Education, v. 72, p. 413-434, 2016a.

MATEOS CORTÉS, Laura Selene; DIETZ, Gunther. How Intercultural is an 'Intercultural University’? Revista Lusófona de Educação, v. 31, p. 125-143, 2016.

MCCOWAN, Tristan. Forging radical alternatives in higher education: the case of Brazil. Other Education, v. 5, n. 2, p. 196-220, 2016.

MCCOWAN, Tristan. Higher education, unbundling and the end of the university as we know it. Oxford Review of Education, v. 43, n. 6, p. 733-748, 2017.

MCCOWAN, Tristan. Higher Education for and beyond the Sustainable Development Goals. London: Palgrave Macmillan, 2019.

MORPHEW, Christopher C.; HUISMAN, Jeroen. Using institutional theory to reframe research on academic drift. Higher Education in Europe, v. 27, n. 4, p. 491-506, 2002.

SANTHAKUMAR, V. et al. Indigenization of Higher Education: Trends in India, Latin America and New Zealand. In: UNIVERSITY PRACTICE CONNECT. Karnataka, India: Azim Premji University, 2020. Available at: https://practiceconnect.azimpremjiuniversity.edu.in/indigenization-of-higher-education-trendsin-india-latin-america-and-new-zealand/. Accessed on: October 25th, 2021.

SANTOS, Boaventura de Sousa. Decolonising the University: The Challenge of Deep Cognitive Justice. Newcastle: Cambridge Scholars Publishing, 2017.

SANTOS, Boaventura de Sousa. The End of the Cognitive Empire: The Coming of Age of Epistemologies of the South. Durham: Duke University Press, 2018.

SILOVA, Iveta. Comparative education as sympoiesis: facing the Anthropocene. Comparative Education Review, Chicago, v. 65, n. 4, $24^{\text {th }}$ May, 2021. 
UNESCO. Report of the Global Independent Expert Group on the Universities and the 2030 Agenda. Paris: UNESCO, 2021.

UNESCO. IIEP. Six ways to ensure higher education leaves no one behind (Policy Paper 30). Paris: UNESCO, 2017.

WILLIAMSON, Ben. The hidden architecture of higher education: Building a big data infrastructure for the 'smarter university'. International Journal of Educational Technology in Higher Education, v. 15, n. 1, p. 1-26, 2018.

Tristan McCowan é Professor de Educação internacional no Institute of Education, University College London. Seu trabalho está concentrado em ensino superior e desenvolvimento internacional e seu livro mais recente é Higher Institution for and beyond the Sustainable Development Goals (Palgrave Macmillan, 2019).

ORCID: https://orcid.org/0000-0002-0710-7519

E-mail: t.mccowan@ucl.ac.uk

Gunther Dietz é Professor em Estudos Interculturais na Universidad Veracruzana, México, onde trabalha em etnicidade, interculturalidade e educação intercultural/interreligiosa.

ORCID: https://orcid.org/0000-0002-1487-2673

E-mail: guntherdietz@gmail.com

Editor-responsável: Luís Armando Gandin

Este é um artigo de acesso aberto distribuído sob os termos de uma Licença Creative Commons Atribuição 4.0 Internacional. Disponível em: <http:// creativecommons.org/licenses/by/4.0>. 